\title{
Functional Phenotyping of the Maternal Albumin Turnover in the Mouse Placenta by Dynamic Contrast-Enhanced MRI
}

\author{
Vicki Plaks, ${ }^{1}$ Stav Sapoznik, ${ }^{1}$ Elina Berkovitz, ${ }^{2}$ Rebecca Haffner-Krausz, ${ }^{2}$ Nava Dekel, ${ }^{1}$ \\ Alon Harmelin, ${ }^{2}$ Michal Neeman ${ }^{1}$ \\ ${ }^{1}$ Biological Regulation, The Weizmann Institute of Science, Rehovot, 76100 Israel \\ ${ }^{2}$ Veterinary Resources, The Weizmann Institute of Science, Rehovot, 76100 Israel
}

\begin{abstract}
Purpose: The purpose of this study was to develop a tool for functional phenotyping of the maternal circulation in the mouse placenta.

Procedures: In utero macromolecular dynamic contrast-enhanced magnetic resonance imaging (DCE-MRI) was performed on embryonic day 10.5 (E10.5), E13.5, and E18.5. Fluorescence analysis was also used for validation of the results.

Results: The initial rate of contrast enhancement revealed an increased maternal blood volume fraction as the pregnancy progressed. Serial imaging of E10.5 and E13.5 placentas revealed a loss of contrast enhancement due to phagocytic uptake. A key application of macromolecular DCE-MRI would be to follow mouse pregnancies during fetal and placental manipulation including embryo transfer, tetraploid complementation, and fetal resorptions. We were able to resolve strain differences in ICR outbred mice carrying both ICR and C57BI/6J embryos and to differentiate in utero resorptions from functional placentas.

Conclusions: Our results highlight the importance of the functional in utero analysis of placental vascularization in physiological phenotyping of transgenic mice and suggest MRI, particularly macromolecular DCE-MRI, as a non-invasive tool for the analysis of the placenta.
\end{abstract}

Keywords: Placenta, Fetus, MRI, Pregnancy, DCE-MRI, Macromolecular contrast material, Mouse model

\section{Introduction}

$\mathrm{T}$ he mouse is widely used as a model animal for the identification of mammalian genes and analysis of their role in both health and disease. The genomic and physiological similarities between mice and humans lead to the use

Significance: Functional phenotyping of the placenta is critical for understanding the impact of genetic manipulations on fetal development. Molecular imaging was applied here for noninvasive mapping of the recycling of maternal plasma albumin in the mouse placenta.

Vicki Plaks and Stav Sapoznik contributed equally to this work.

Correspondence to: Michal Neeman; e-mail: michal.neeman@weizmann.ac.il of the mouse model as a research tool to test suggested therapies of human diseases. Taking advantage of the known mouse genomic sequence, a growing scientific effort is currently being made in order to generate knockouts for each of the mouse genes, providing a tool for the identification of their role and function [1]. A variety of additional tools is being exploited for genomic manipulation, including: (1) gene overexpression, (2) knock-in strategies, (3) reporter gene insertions, and (4) generation of conditional transgenic mice, emphasizing the need to study gene specific functions in the context of the specific organ in question. In this respect, tetraploid placenta complementation, in which an embryo can be complemented with a placenta of a different genetic composition, enables the assessment of the role of a 
gene in embryonic versus extra-embryonic tissues (such as the placenta). In addition, tetraploid complementation can be used to prevent in utero death of embryos due to placental malfunction. The method involves aggregation of electrofused tetraploid pre-implantation embryos with embryonic stem cells (or inner cell mass), resulting in the development of the extraembryonic lineages (including the placenta) from the tetraploid embryo and embryonic lineages from the embryonic stem cells. In this way, it is possible to generate an embryo with a specific genetic background supported by extra-embryonic tissues of another genetic background [2, 3, 5].

Side by side with the increased availability of genetically modified mice, an effort is being made to develop methodologies for the phenotypic analysis of newly formed animal models. Basic phenotype screening programs typically include behavioral observation, assessment of developmental lethality, physical examination, and blood tests [1]. However, a more detailed analysis is needed in order to fully understand the role and function of the gene of interest. Non-invasive analyses which do not hamper the viability of the mouse model in question bear an obvious advantage.

The aim of this study was to develop a novel tool for the phenotypic analysis of mouse development in utero, with emphasis on the placenta. The importance of the mammalian placenta is derived from the fundamental role it has in exchange of respiratory gases, nutrients, and wastes between the maternal and fetal vascular systems [6-10]. The insight on the maternal and fetal circulation of the mouse placenta was enhanced by an elegant application of vascular casts [11]. The analogy between the structure and function of the mouse and human placenta was carefully examined by histopathology, suggesting multiple similarities in structure and function, despite specific differences [12].

We previously applied blood oxygen level-dependent magnetic resonance imaging (MRI) to non-invasively monitor hypoxia induced by cloprostenol, a prostaglandin $\mathrm{F}$ (2alpha) analog, in the rat embryo placental unit [13]. Additionally, macromolecular dynamic contrast-enhanced (DCE) MRI using biotin-bovine serum albumin (BSA)-Gd-DTPA was applied for the in vivo characterization of vascular function in tumors $[14,15]$, in ovarian grafts [16], and during embryo implantation [17]. In these studies, biotin-BSA-Gd-DTPA selectively extravasated from areas where the vessel permeability was high, such as tumors, embryo implantation sites, and ovaries, and allowed non-invasive imaging of these areas of interest. Biotin-BSA-Gd-DTPA provides a complementary ability for the validation of the biodistribution of the MRI contrast agent in histological sections by labeling with avidin-Texas red. However, in contrast to low molecular weight gadolinium chelates, albumin does not cross the placenta barrier in normal situations and, thus, the analysis of maternal circulation in the placenta is not confounded by transfer to the fetal circulation, as is seen with Gd-DTPA [18, 19].

In this study, we evaluated in utero normal placentas at different developmental stages and resolved strain-specific differences. At the first stage of analysis (E10.5), the chorioallantoic placenta had reached its basic three-layer structure. The placenta continues its expansion and rearrangement throughout pregnancy (E13.5) and supplies the needs of the embryo until the end of the pregnancy (E18.5-19.5). The materno-placental vascular bed was characterized in manipulated mouse embryos (including transfer of normal and tetraploid-complemented embryos to foster dams), differentiating normally functioning placentas from those undergoing resorption. Since the macromolecular DCE-MRI and the fluorescence analysis used here are enabled by the blood-borne transport of the albumin-based contrast material, these methods provide information on the endogenous flux of maternal blood through the placentas examined. We suggest that macromolecular DCE-MRI is a valuable tool for the functional in utero phenotypic evaluation of the materno-placental circulation in wild-type and genetically modified mice.

\section{Materials and Methods}

All animal experiments were approved by the Weizmann Institutional Animal Care and Use Committee.

\section{In Vivo Contrast-Enhanced MRI Studies}

Pregnant female mice (ICR outbred mice, Harlan, Israel; 710 weeks old) were analyzed by MRI on E10.5 $(n=2$, total of 15 embryos, vaginal plug $=\mathrm{E} 0.5), \mathrm{E} 13.5(n=2$, total of 24 embryos $)$, and E18.5 ( $n=3$, total of 21 embryos). Different groups of mice were used at each stage of the pregnancy for the analysis. Female ICR mice carrying transferred embryos, $(n=7$ ICR; C57B1/6J (B6) with native (four embryos) or tetraploid placentas (five embryos)), were analyzed on E18.5 of pregnancy.

MRI experiments were performed on a horizontal 4.7 T Bruker Biospec spectrometer (Karlsruhe, Germany) using a whole-body birdcage rf coil, with a diameter of $7.5 \mathrm{~cm}$. No quadrature detection was applied. Mice were anesthetized with intraperitoneal injection of $75 \mathrm{mg} / \mathrm{kg}$ ketamine (Ketaset; Fort Dodge Laboratories, Fort Dodge, IA, USA) and $3 \mathrm{mg} / \mathrm{kg}$ xylazine (XYL-M 2\%; VMD, Arendonk, Belgium) followed by the subcutaneous addition of about $30 \%$ of the initial dose in order to prolong the anesthesia. Anesthetized mice were placed in prone position on a polymethylmethacrylate (Perspex) board so that the abdomen was at the center of the coil, immobilized using adhesive tape and covered with a cloth blanket (to retain body temperature). The magnet room was maintained at $25^{\circ} \mathrm{C}$.

The BSA-based macromolecular contrast material, biotin-BSAGd-DTPA (approximate MW is $82 \mathrm{kDa}$, relaxivity $R_{1}=135 \mathrm{mM}^{-1} \mathrm{~s}^{-1}$ per albumin), was prepared as reported previously $[15,20]$ and injected through a tail vein catheter as bolus at $12 \mathrm{mg} /$ mouse in $0.2 \mathrm{~mL}$ of phosphate-buffered saline (PBS).

A series of variable flip angle (FA), precontrast, $\mathrm{T}_{1}$-weighted threedimensional gradient echo (3D-GE) images was acquired to determine the endogenous precontrast $R_{1}$ (pulse FA $15^{\circ}, 5^{\circ}, 30^{\circ}, 50^{\circ}, 70^{\circ}$; repetition time (TR) $10 \mathrm{~ms}$; echo time (TE) $3.6 \mathrm{~ms}$; two averages, spectral width $50,000 \mathrm{~Hz}, \mathrm{FOV}=5 \times 5 \times 5 \mathrm{~cm}$, matrix $128 \times 128 \times 64$, zero filled to $128 \times 128 \times 128$, voxel resolution $0.39 \mathrm{~mm}^{3}, 2 \operatorname{min~} 43 \mathrm{~s}$ between each image). For dynamic post-contrast imaging, consecutive $\mathrm{T}_{1}$-weighted 3D-GE images were acquired from the time of biotinBSA-Gd-DTPA administration up to $13.58 \mathrm{~min}$ (pulse $\mathrm{FA}=15^{\circ}$ and 
all other parameters are as stated above; five scans post-contrast were collected). Fetal anatomy was derived from multi-slice spin echo images (TR 2,000 ms; TE $11 \mathrm{~ms}$, two averages, spectral width 50,000, matrix $128 \times 128$, in-plane resolution $0.39 \mathrm{~mm}^{2}$, slice thickness $1 \mathrm{~mm}$, interslice distance $1.2 \mathrm{~mm}, 13$ slices).

MR signal intensity and the precontrast $R_{1}$ values $\left(R_{1 \text { pre, }}\right.$ derived from the variable flip angle scans; Eq. 1) were used to derive the postcontrast $R_{1}$ values $\left(R_{1 \text { post }} ;\right.$ Eq. 2$)$ and the contrast material concentration for each time point $\left(C_{\mathrm{t}}\right.$; Eq. 3$)$, as reported previously [17], using Paravision (Paravision 4, Bruker, Rheinstetten, Germany) and Matlab (Matlab 7.6.0 (R2008a), The Math Works Inc., Natik, MA, USA).

$$
\begin{gathered}
I=\frac{M_{0} \sin \alpha\left(1-e^{-\mathrm{TR} \cdot R_{1 \mathrm{pre}}}\right)}{1-\cos \alpha \cdot e^{-\mathrm{TR} \cdot R_{1 \mathrm{pre}}}} \\
\frac{I_{\mathrm{pre}}}{I_{\mathrm{post}}}=\frac{M_{0} \sin \alpha\left(1-e^{-\mathrm{TR} \cdot R_{1 \mathrm{pre}}}\right) /\left(1-\cos \alpha \cdot e^{-\mathrm{TR} \cdot R_{1 \mathrm{pre}}}\right)}{M_{0} \sin \alpha\left(1-e^{-\mathrm{TR} \cdot R_{\mathrm{ppost}}}\right) /\left(1-\cos \alpha \cdot e^{-\mathrm{TR} \cdot R_{1 \mathrm{post}}}\right)} \\
C_{\mathrm{t}}=[\mathrm{biotin}-\mathrm{BSA}-\mathrm{GdDTPA}] \\
=r_{1}^{-1}\left(R_{1 \mathrm{post}}-R_{1 \mathrm{pre}}\right)
\end{gathered}
$$

where $I$ is the signal intensity for each flip angle $\alpha$ and $R_{1}$ is the specific relaxivity of biotin-BSA-Gd-DTPA $\left(135 \mathrm{mM}^{-1} \mathrm{~s}^{-1}\right.$ per albumin). The analysis is based on the assumption that water exchange between the fetal and maternal circulation is fast. The concentration changes were fit by linear extrapolation (Eq. 4):

$$
\frac{C_{\mathrm{t}}}{C_{\text {blood }}}=\frac{C_{0}}{C_{\text {blood }}}+\mathrm{ROE} \cdot t
$$

where the initial enhancement was derived from the ratio of extrapolated contrast material concentration at time $=0$ relative to the concentration in the vena cava $\left(C_{0} / C_{\text {blood}}\right.$; initial enhancement). The rate of change in contrast material concentration was scaled to the concentration in the vena cava $(\mathrm{ROE}=$ rate of enhancement with units of $\mathrm{min}^{-1}$ ). Values were calculated from manually selected regions of interest (ROI); the ROI was chosen from the inner part of each enhanced placenta using the third time point postcontrast. For some of the placentas (in which there were movements of the fetuses), the ROI was manually marked for each scan. Fig. 2 shows a representative analysis of MRI data and ROI selection. The initial enhancement gives an indication to the initial blood volume that fills the materno-placental blood spaces, while the rate of enhancement over time gives an indication on the dynamics of albumin turnover in the placenta.

Since on E10.5, in contrast with E13.5 and E18.5, the decidua is still a significant part of the placenta, it was taken into account when the ROI was chosen for quantification. On E13.5 and specifically on E18.5, the decidua is significantly smaller and less vascularized as compared to the labyrinth. By choosing an ROI within the enhanced region of the placenta, we were able to mostly avoid the deciduas and concentrate on the labyrinth at E13.5 and E18.5. Placental volume was calculated by quantifying the number of voxels from all the slices in which the placenta appeared in the 3D-MRI data. Fetal length was calculated from the mid-coronal or mid-sagittal MR images.
Maximal intensity projections and surface projections were assembled using NIH image and Paravision, respectively.

\section{Histology, Histochemistry, and Fluorescence Microscopy}

Prior to animal euthanasia, BSA labeled with 6-carboxy-X-rhodamine (BSA-ROX) was injected via the tail vein catheter previously used for MRI for the histological detection of functional blood vessels, as reported previously [20]. In a separate set of experiments, carboxyfluorescein (BSA-FAM) was administered by intravenous (i.v.) injection and the animals were euthanized by cervical dislocation either 3.5 or 13.5 min after fluorescent contrast material administration and placentas were retrieved for further analysis. A macro view of fetuses and placentas was acquired using regular and fluorescent light (Olympus SZX 12 microscope, Center Valley, PA, USA, equipped with DP50 camera). Placentas were fixed in Carnoy mixture, embedded in paraffin, and sectioned serially at $4-\mu \mathrm{m}$ thickness. The fluorescent contrast material was detected using a fluorescent microscope (Zeiss Axioscope II, Yena, Germany, Simple PCI software). The percentage of fluorescent staining coverage in each field of view was calculated using ImageJ software. Biotin-BSA-GdDTPA was detected by staining of sections with avidin-Texas red (Invitrogen, Carlsbad, CA, USA) [20]. Cell nuclei were fluorescently stained with 4',6-diamidino-2phenylindole (DAPI; Vector Laboratories, Burlingame, CA, USA).

\section{Embryo Transfer and Tetraploid Complementation/Rescue}

Tetraploid aggregation is an important tool frequently used for the detection and prevention of in utero lethality of genetically modified mice, which is secondary to defects in extra-embryonic tissues such as the placenta. By doubling the ploidity at the two-cell stage through electrofusion, tetraploid embryos cannot develop but can still contribute to the extra-embryonic tissue when aggregated with the inner cell mass (ICM) of diploid embryos (isolated at the blastocyst stage) [2-5]. The results of this manipulation are embryos of one genetic background, supported by placentas of a different genetic background. In the experiments reported here, GFP-expressing embryos provided the tetraploid placenta for the B6 embryos.

\section{Embryo Collection and Culture}

For superovulation, 3-week-old ICR females were injected intraperitoneally with $5 \mathrm{IU}$ of pregnant mare serum gonadotropin (PMSG) and $46 \mathrm{~h}$ later with $5 \mathrm{IU}$ of human chorionic gonadotropin (hCG). After hCG injection, females were mated with B5/EGFP males [21]. The superovulated females were euthanized 46-48 h after hCG (E1.5), and late, two-cell embryos were collected by flushing the oviduct.

Five- to 6-week-old B6 females were superovulated with 5 IU of PMSG and $10 \mathrm{IU}$ of hCG and mated with B6 males. Females were euthanized either on E2.5, and eight-cell embryos were isolated for aggregation, or E3.5, when blastocysts were isolated for immunosurgery.

\section{Immunosurgery}

The trophectoderm layer of the diploid blastocysts (E3.5) was removed by immunosurgery, thus isolating the ICM as previously described [22]. 


\section{Production of Tetraploid Embryos}

Two-cell embryos underwent electrofusion. Following a short pulse, embryos were transferred to the incubator. After $24 \mathrm{~h}$, compacting four-cell-stage embryos were used for aggregation [23].

\section{Aggregation of Tetraploid Embryo and Isolated Inner Cell Masses}

After the removal of the zona pellucida, single, isolated ICM was sandwiched between two tetraploid embryos in an aggregation plate. After 24-h incubation, blastocysts were transferred to the uterus of E2.5 pseudopregnant ICR female, at eight to ten blastocysts per uterine horn.

In the experiments in which embryo transfer only was performed, E3.5 embryos were transferred to the uterus of E2.5 pseudopregnant ICR female, at eight to ten blastocysts per uterine horn.

\section{Statistical Analysis}

A two-tailed unpaired Student's $t$-test was applied for the analysis of significance of the MRI and histological data. The data are considered significant when $P<0.05$. Data are presented as mean value \pm standard error of the mean. Two-way ANOVA analysis was applied for the fluorescence rate of enhancement.

\section{Results}

\section{In Utero MRI Evaluation of Placental Size and Maternal Blood Circulation}

Pregnant mice were studied by macromolecular DCE-MRI at three different stages (Fig. 1): E10.5 $(n=2,15$ placentas, dropout rate of 35\%), E13.5 ( $n=2,24$ placentas, dropout rate of $4 \%)$ and E18.5 $(n=3,21$ placentas, dropout rate of $8 \%)$. All placentas that were discarded from the analysis showed bad fit, predominantly due to movements of the embryos, which were more intense at E10.5.

Maximal intensity projections enabled the detection of the maternal blood spaces including the embryonic placentas (Fig. 1a-f). Placental volume (Fig. 1g) showed a threefold increase from E10.5 $(0.01 \pm 0.005 \mathrm{~mL})$ to E13.5 $(0.03 \pm$ $\left.0.0006 \mathrm{~mL}, P=1.17 \times 10^{-12}\right)$ and a threefold increase from E13.5 to E18.5 $\left(0.09 \pm 0.01 \mathrm{~mL}, P=3.9 \times 10^{-12}\right)$.

The initial contrast enhancement in the placenta (Fig. 1h) revealed no significant change in the maternal blood volume fraction between E10.5 $(0.2 \pm 0.03)$ and E13.5 $(0.15 \pm 0.02, P=$ $0.13)$. However, a considerable increase in the initial enhancement (Fig. 1h), by 3.3-fold, was observed at E18.5 compared to E13.5 $(0.5 \pm 0.15, \mathrm{P}=0.000016)$. These data show that, at midpregnancy, the increase in total placental volume precedes the increase in maternal blood volume fraction.

Follow-up of the dynamics of contrast enhancement for $13.5 \mathrm{~min}$ was used for the derivation of the rate of change in contrast material concentration over time (Fig. 1i). At the early stage of placental development (E10.5), a negative rate of enhancement $\left(-0.007 \pm 0.007 \mathrm{~min}^{-1}\right)$ was observed, rather than accumulation; there was a rapid rate of clearance of albumin from the maternal circulation of the placenta which was faster than the rate at which biotin-BSA-Gd-DTPA was cleared from the circulation (Fig. 2). This clearance was attenuated at E13.5 $\left(-0.0016 \pm 0.0008 \mathrm{~min}^{-1}\right)$. Towards the end of pregnancy (E18.5), the contrast material accumulated slowly in the materno-placental blood spaces due to the slow exchange of maternal blood in the placenta resulting in a positive rate of enhancement $\left(0.005 \pm 0.0049 \mathrm{~min}^{-1}\right)$. A significant change was observed between all time points detected $(P=0.01$ for E10.5 and E13.5 and $P=0.004$ for E13.5 and E18.5). No enhancement could be detected in the embryos at any stage.

\section{Histological Analysis of Placental Initial Enhancement and Rate of Enhancement Over Time}

The MRI data was validated using the analogous fluorescent macromolecular contrast agent BSA-FAM. Pregnant ICR mice at different stages of pregnancy $[\mathrm{E} 10.5 \quad(n=3$, nine embryos), E13.5 ( $n=3,13$ embryos) and E18.5 $(n=2$, seven embryos)] were administered with BSA-FAM (5 $\mathrm{mg}$ in PBS i.v.) and euthanized either 3.5 or $13.5 \mathrm{~min}$ later. Placentas were fixed and sectioned serially. The data in Fig. 3 show that the contrast material is retained in the maternal blood spaces with no transfer of the contrast media to the fetal circulation. This indicates that our analysis is of the maternal part of placental circulation and that the positive rate of contrast agent accumulation at E18.5 does not represent vascular permeability.

Based on this finding, we analyzed the fluorescent data for changes in the distribution of contrast media that can explain the clearance observed in the early-stage placenta versus the accumulation observed in the late-stage placenta. Analysis of the percentage of fluorescent area coverage in each field of view retrieved from animals euthanized $3.5 \mathrm{~min}$ post-injection (Fig. 3a-c) enabled a calculation of the initial rate of enhancement in placenta. A 1.4-fold increase in the initial enhancement (Fig. 3g) in the labyrinth was observed between E10.5 (11.33 $\pm 1.68 \%)$ and E13.5 $(15.5 \pm 4.18 \%, P=$ $0.015)$ as well as between E13.5 and E18.5 (20.77 $\pm 5.05 \%$, $P=0.02$ ). However, in corroboration with the MRI data in Fig. 1, when the decidua blood pools at E10.5 were taken into account (E10.5 dec + lab, 15.08 \pm 1.18$)$, there was no significant difference between the initial enhancement at E10.5 and E13.5 $(P=0.13)$.

Comparison of the fluorescent area at 13.5 min (Fig. 3d-f) and at 3.5 min after administration of BSA-FAM enabled the calculation of the rate of enhancement. The calculation was done by subtracting the mean percentage area coverage of BSA-FAM positive blood vessels at $3.5 \mathrm{~min}$ from the mean area at $13.5 \mathrm{~min}$. A similar trend was observed as shown above by MRI. Rapid clearance of the fluorescent contrast agent was observed at E10.5 (mean $-4.37 \%$ ), compared with a slower 
$\mathrm{E} 10.5$
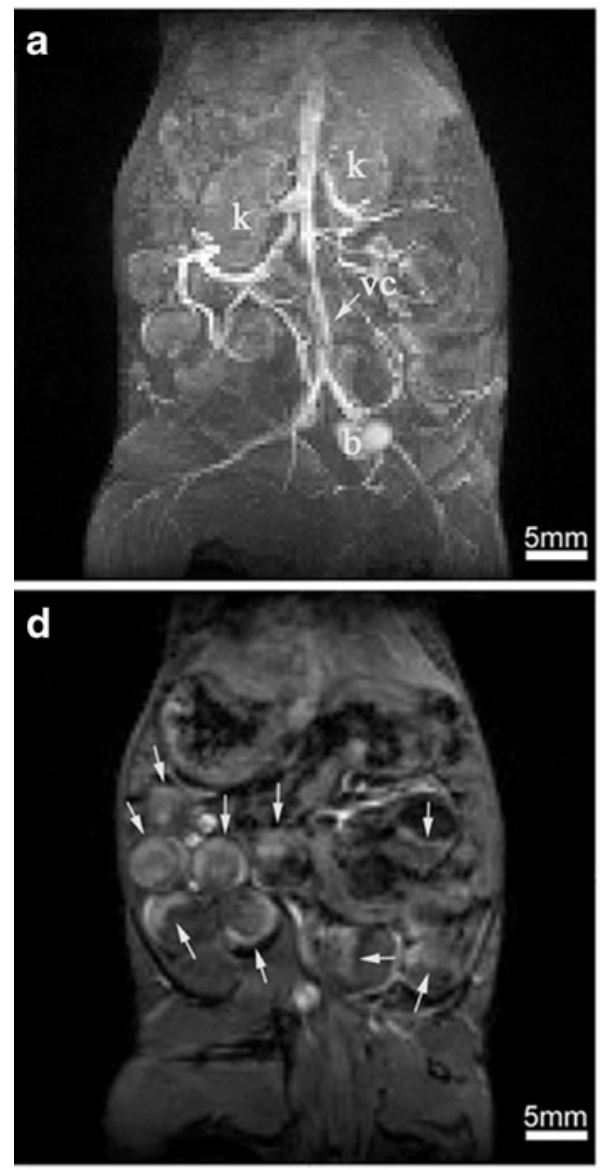

g

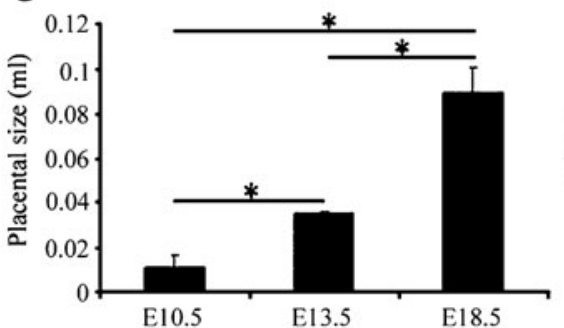

E13.5
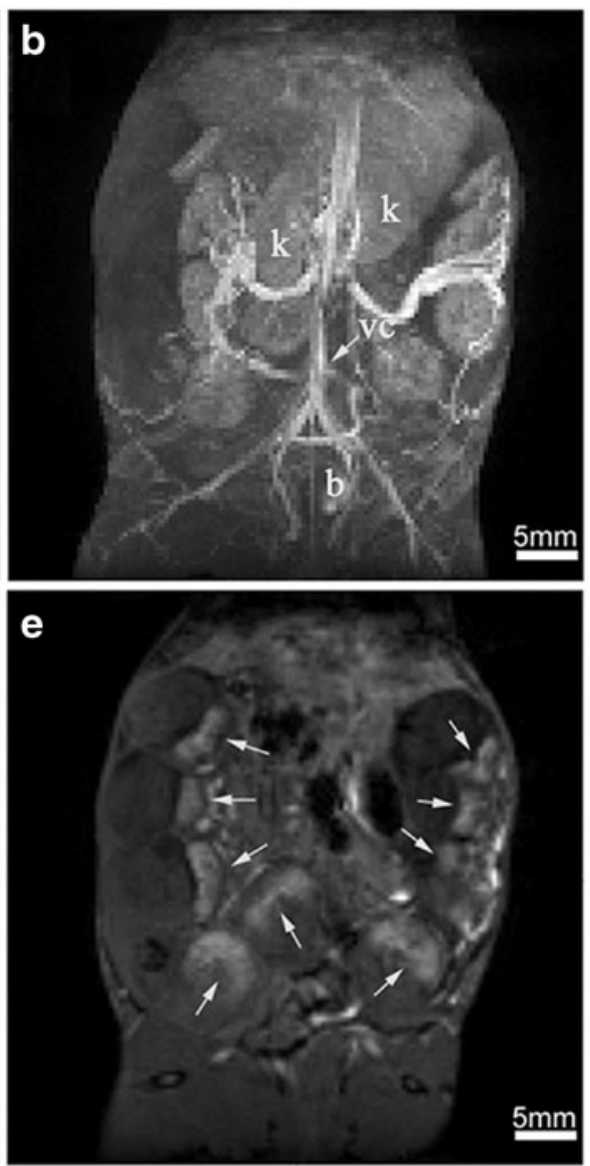

h

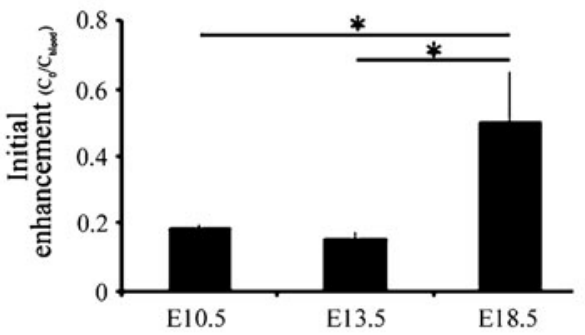

E18.5
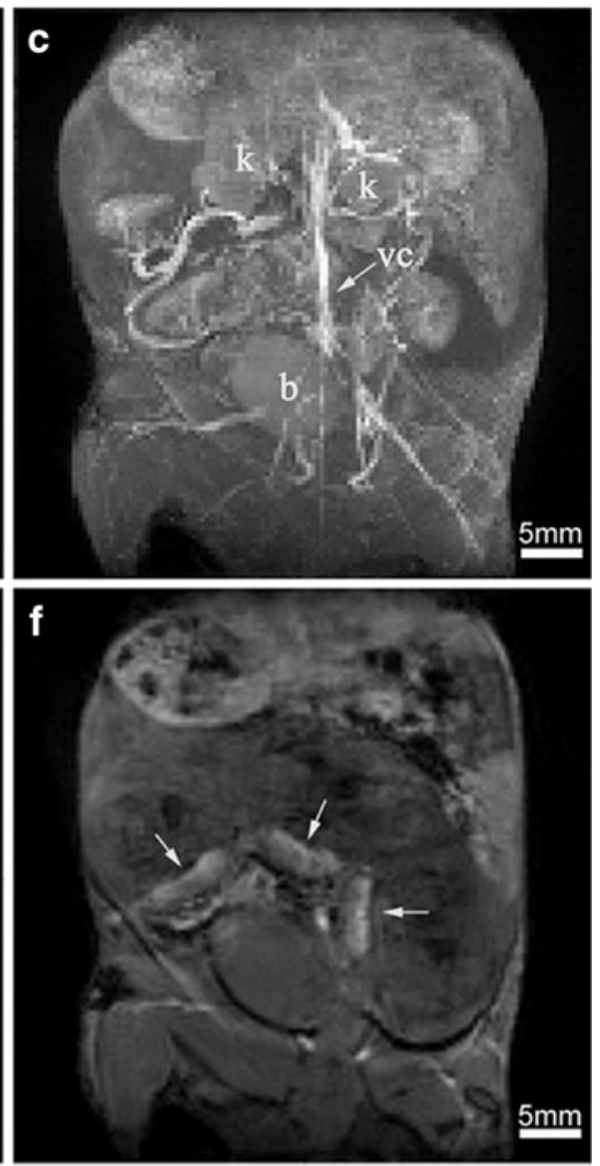

i

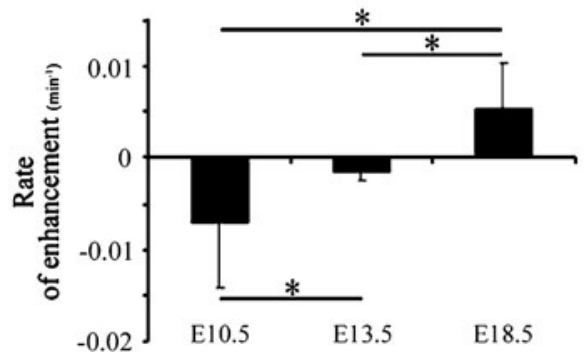

Fig. 1. In utero MRI evaluation of placental size, initial enhancement, and rate of enhancement during different embryonic stages (E10.5, E13.5, E18.5). a-c Maximal intensity projections of pregnant ICR mice, tail vein injected with biotin-BSA-GdDTPA at E10.5, E13.5, or E18.5 respectively. $K$, kidney; $B$, bladder; VC, vena cava. d-f Surface projections of a-c, respectively. Placentas are indicated with an arrow. $\mathbf{g}$ Calculation of the placental size (in $\mathrm{mL}$ ) at different developmental stages. $\mathbf{h}$ Calculation of the initial enhancement in the placenta (calibrated to the vena cava) at different developmental stages. $\mathbf{i}$ Calculation of placental rate of enhancement at different developmental stages.

clearance at E13.5 (mean -1.77\%). At E18.5, no clearance was detected (mean 2.43\%) and the contrast agent accumulated slowly within the materno-placental blood spaces (Fig. 3h, two-way ANOVA, $F_{2,25}=10.87, P=7.1 \times 10^{-9}$, post-hoc analysis: E10.5 vs. E13.5: $P=2.45 \times 10^{-4}$; E10.5 vs. E18.5: $P=$ $8.47 \times 10^{-9}$; E13.5 vs. E18.5: $P=2.29 \times 10^{-4}$ ).

Hematoxylin and eosin (H\&E)-stained placentas of the various developmental stages show the development of the maternal blood spaces in the labyrinth and decidua (Fig. 3i-k). Branching morphogenesis occurs at the chorioallantoic interface specifically at E13.5 as compared to the smaller size and shallow interdigitation of the maternal blood spaces on E10.5. However, at E10.5, the area of the blood pools in the decidua is relatively large to that of the labyrinth: the labyrinth is $46 \%$ the size of the decidua. At E13.5 and E18.5, the decidua is significantly smaller and less vascularized as compared to the labyrinth; the labyrinth is 5.75 times the size of the decidua at E13.5 and 14.5 times at E18.5. By E18.5, the labyrinth had reached its full size and largest volume fraction [24].

Since the MR contrast material enhances the maternal side of the placenta, i.e., the maternal blood spaces, at E10.5, the MRI data in Fig. 1 represents both the labyrinth and the 
$\mathbf{a}$

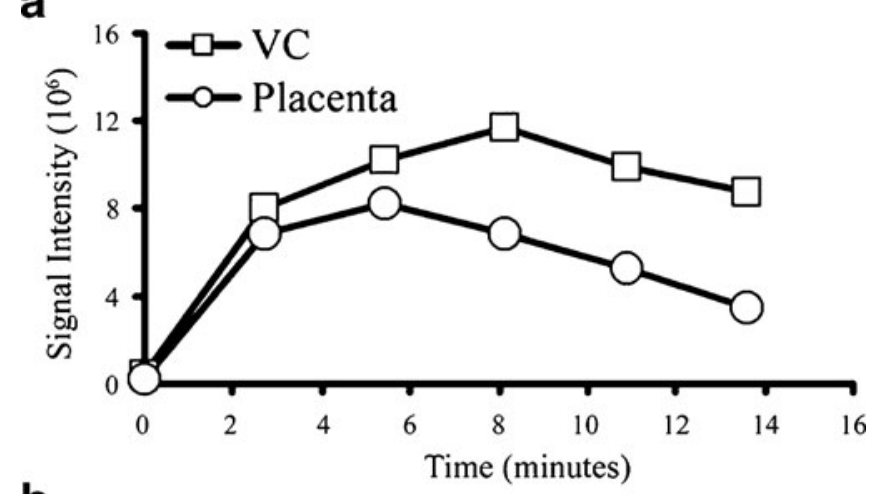

b

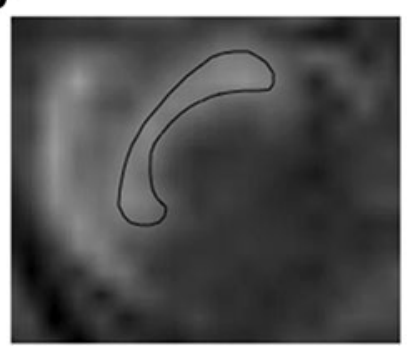

Fig. 2. MRI quantification analysis and ROI selection. a Representative analysis of signal intensity over time in E10.5 placenta and maternal vena cava before (time $=0$ ) and following injection of biotin-BSA-Gd-DTPA. b Surface projection of a placenta marked with a ROI.

decidua blood pools, as exemplified by the initial enhancement calculated both for the labyrinth and the decidua relative to the area of each compartment. The rate of enhancement at E10.5 is similar for both compartments, as also seen in the MRI data in Fig. 1. At E13.5 and E18.5, the MRI data in Fig. 1 represents only the maternal blood in the labyrinth, as illustrated by the size of the labyrinth in the histology.

The rapid clearance of the MRI and fluorescence contrast materials from the maternal circulation at E10.5, E13.5, and E18.5 was examined (Fig. 31-n). The biotin-BSA-GdDTPA was allowed to circulate for $50 \mathrm{~min}$ and, just before animal euthanasia, BSA-FAM was injected. As can be seen, both the endothelial cells in E10.5 decidua as well as the multicellular syncytiotrophoblasts and the E13.5 labyrinth have internalized biotin-BSA-Gd-DTPA (stained with avidin-Texas red) while the BSA-FAM (green) is still located within the vessel lumen. At E18.5, the early (red) and late (green) contrast materials co-localize (yellow) within the maternal blood spaces, implying an accumulation of the contrast material within this compartment. Therefore, the internalization of albumin at early developmental stages is partly due to the contrast material uptake from the placental blood spaces. This uptake can also be observed at the later stage (E18.5), but it is confounded by the gradual accumulation of the contrast material in the maternal blood spaces.

\section{Resolving Strain-Specific Differences Using MRI}

In order to elaborate the capability of macromolecular DCEMRI for the evaluation of developmental changes in utero, we examined strain-related differences using embryo transfer experimentation (Fig. 4). B6 and ICR embryos were segregated between the two uterine horns, so each strain was transferred to a different uterine horn of a pseudopregnant ICR mouse ( $n=2$, eight B6 embryos, 12 ICR embryos).

Fig. 3. Histological analysis of maternal albumin turnover in the mouse placenta. Fluorescent images of placental labyrinth histological sections retrieved from E10.5, E13.5, and E18.5 embryos, respectively. For E10.5, the inserts are of the decidua blood pools (at the same magnification as the labyrinth) (a-f). a-c Placentas were retrieved for histology 3.5 min following tail vein injection of BSA-FAM to the pregnant mother. Blue, DAPI. Green, BSA-FAM. d-f Placentas were retrieved for histology $13.5 \mathrm{~min}$ following tail vein injection of BSA-FAM to the pregnant mother. Blue, nuclear staining with DAPI. Green, BSA-FAM. g Calculation of the initial enhancement inside the placenta at different developmental stages. $\mathbf{h}$ Calculation of the placental rate of enhancement by the fluorescent contrast agent at different developmental stages. Please note that, for E10.5, the histograms show the calculations for the labyrinth (lab) and decidua blood pools $(d e c)$ separately and the combined values of the two compartments $(d e c+l a b)$. i-k H\&E-stained placentas of the respected developmental stages exemplify the development of the maternal blood spaces in the labyrinth (lab) and decidua blood pools (dec). I-n Placentas were retrieved $50 \mathrm{~min}$ post-injection of biotin-BSA-GdDTPA via a tail vein (stained with avidin-Texas red) which was followed by BSA-FAM (green) injected through the same catheter 3.5 min prior to animal euthanization. Blue, DAPI. Arrows, uptake of biotin-BSA-Gd-DTPA into intracellular vacuoles detected using avidin-Texas red. 
E10.5
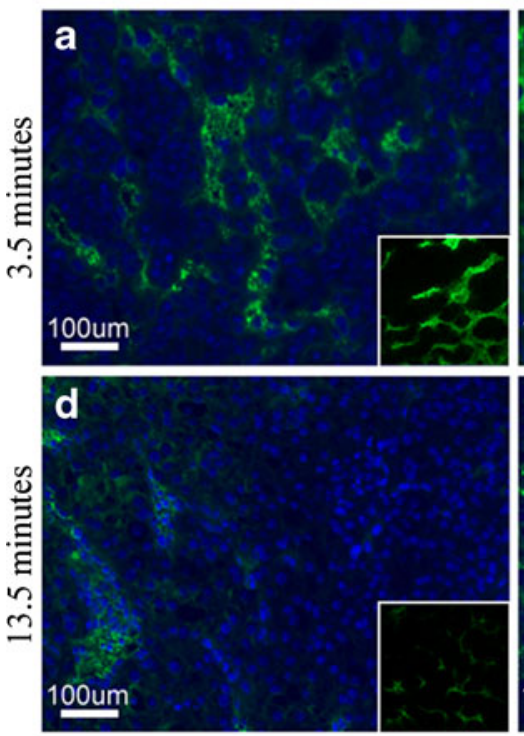

g

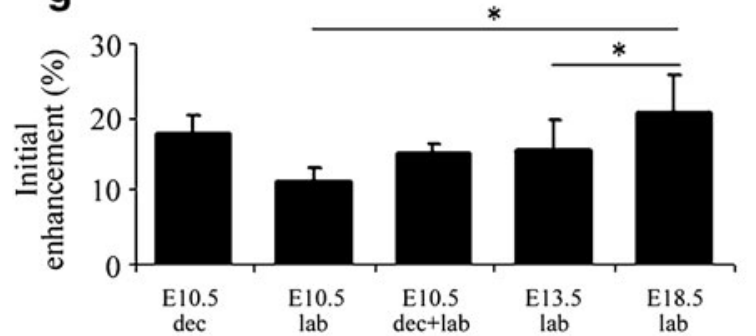

E13.5
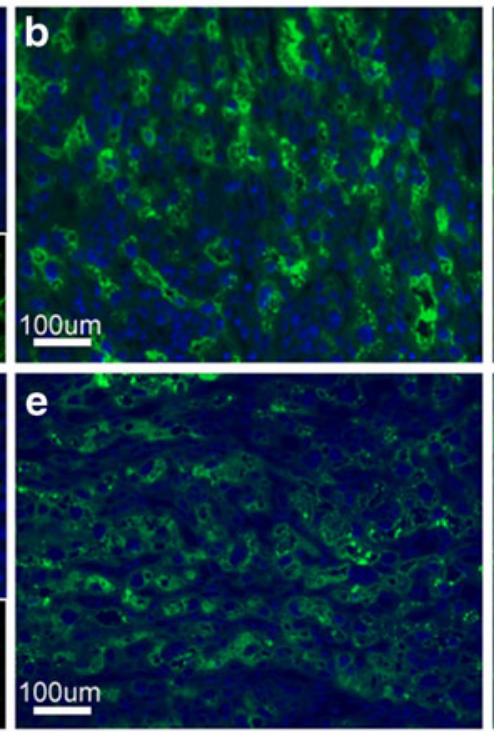

E18.5
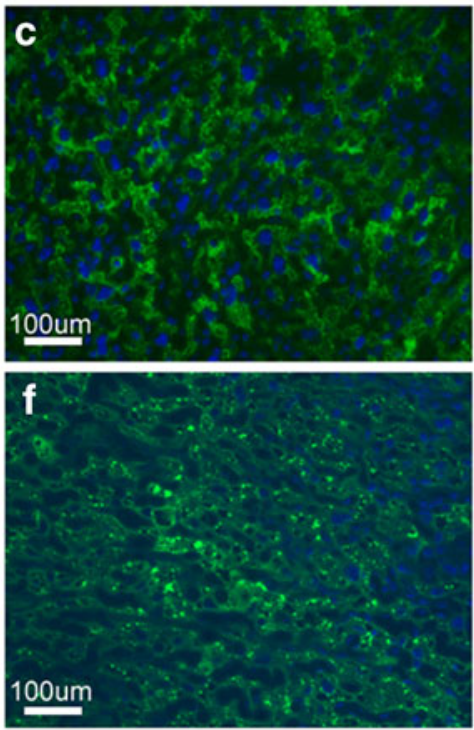

h
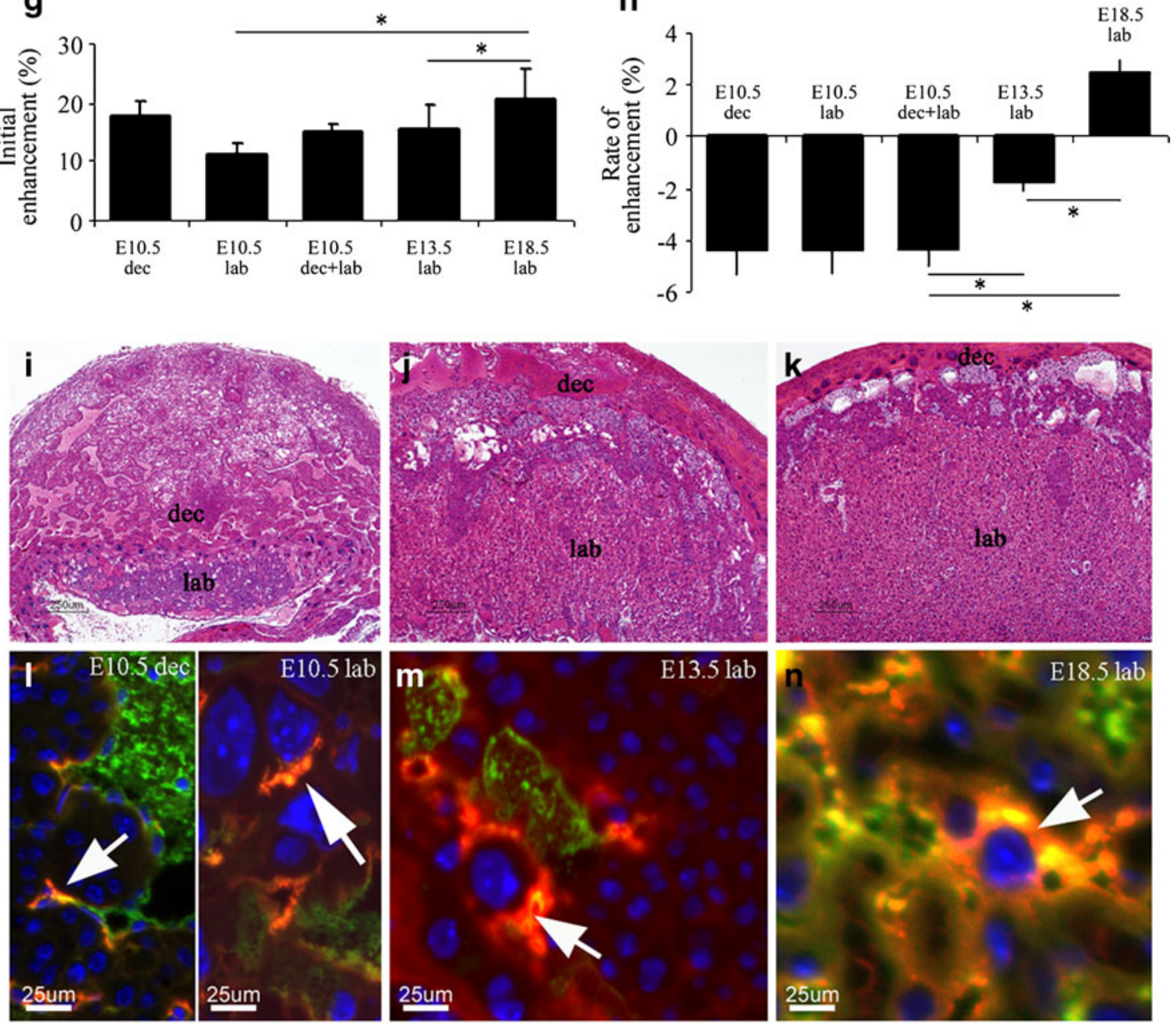

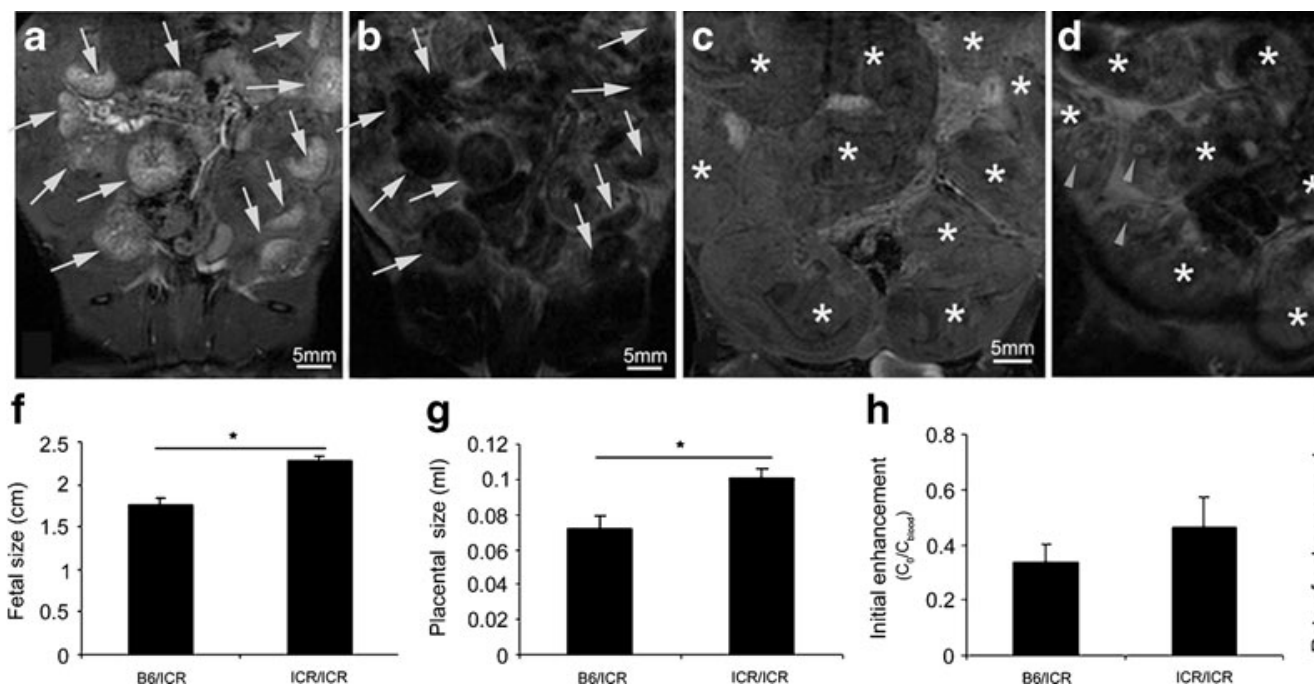
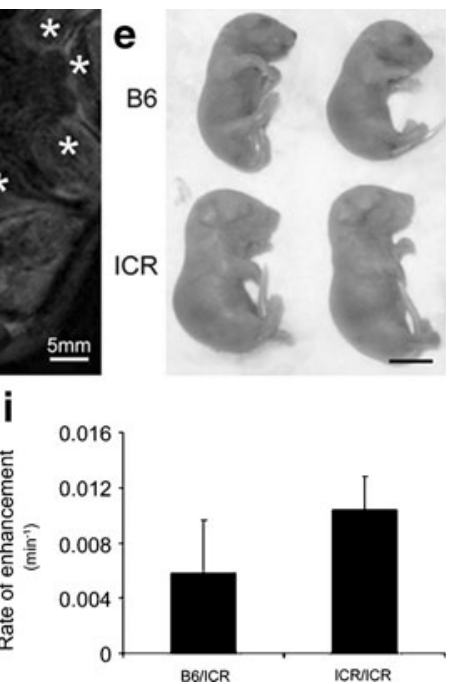

Fig. 4. Resolving strain differences using MRI. a, c Surface projections of a pregnant ICR mouse carrying E18.5 B6 and ICR transferred embryos, tail vein injected with biotin-BSA-Gd-DTPA. Placentas are indicated with an arrow. Embryos are indicated with an asterisk. $\mathbf{b}, \mathbf{d ~ T}_{2}$-weighted images of $\mathbf{a}$ and $\mathbf{c}$ prior to contrast material injection. Placentas are indicated with an arrow. Embryos are indicated with an asterisk. d Embryos' eyes are indicated with arrowheads. e Ex vivo demonstration of B6 and ICR E18.5 embryos. $\mathbf{f}$ Calculation of the B6 and ICR fetal size (cm). $\mathbf{g}$ Calculation of B6 and ICR placental size (mL). $\mathbf{h}$ Calculation of placental initial enhancement for B6 and ICR placentas. i Calculation of placental rate of enhancement for B6 and ICR placentas.

E18.5 embryos were scanned (Fig. 4a-d) and analysis of fetal and placental size, as well as placental initial enhancement and rate of enhancement, was carried out. Using the MRI data, a significant difference was observed in fetal size (Fig. 4f), as ICR embryos were found to be 1.3-fold larger than B6 embryos $(2.28 \pm 0.05$ and $1.75 \pm 0.09 \mathrm{~cm}$, respectively; $P=$ 0.001). This difference was verified following animal euthanasia and retrieval of the embryos (Fig. 4e). In agreement with the change in fetal size, placental size (Fig. 4g) was found to be significantly larger in ICR $v s$. B6 embryos $(1 \pm 0.06$ and $0.72 \pm$ $0.08 \mathrm{~mL}$, respectively; $P=0.03$ ). Interestingly, a tendency towards higher values in the placental initial and rate of enhancement in the ICR embryos was observed in these analyses (Fig. 4h). Strain-related changes in the initial enhancement (ICR, $0.46 \pm 0.09$; B6, 0.35 $\pm 0.06 ; P=0.36$ ) and rate of enhancement (ICR, $1.04 \pm 0.24 \mathrm{~min}^{-1}$; B6, $0.58 \pm 0.37 \mathrm{~min}^{-1}$; $P=0.09$; Fig. 4i) were not significant.

\section{MRI Detection of Normally Functioning Tetraploid Placentas Versus Placental Vascular Resorptions}

Some genetically modified mouse models and manipulation in mouse embryos lead to death in utero, which could be reflected in impaired placental development. During pregnancy, pathological circumstances may lead to resorption of both the embryo and its placenta. As the process occurs gradually, partially perfused placentas can be found in the uterus even after the embryo was already resorped. We applied ex vivo fluorescence imaging for the validation of the in vivo macromolecular DCE-MRI for the detection and evaluation of placental pathologies.
The different physiological states are exemplified in Fig. 5. Fully vascularized placentas attached to healthy embryos were visualized both by intravenous administration of BSA-ROX to pregnant ICR mice E18.5 (Fig. 5a-d) and by macromolecular DCE-MRI (Fig. 5g). However, a fully resorped unvascularized placenta could not be imaged following BSA-ROX i.v. injection (Fig. 5a, b). Interestingly, a partially perfused placenta attached to a fully resorped embryo could be detected by both fluorescent imaging and

Fig. 5. Macromolecular contrast material detects vascular resorptions. a, b Light microscopy (a) and fluorescence microscopy (b) of the same embryo. BSA-ROX (red) is excluded from a fully resorped placenta (arrows in $\mathbf{a}$ and $\mathbf{b}$ ). c, d Functional placentas of resorped fetuses are perfused. e, $\mathbf{f}$ Tetraploid resorption (left) and a fetus that died in utero and its tetraploid GFP placenta (right). $\mathbf{g}, \mathbf{h}$ MRI detection of biotin-BSA-Gd-DTPA of $\mathbf{c}$ and $\mathbf{d}$ versus $\mathbf{e}$ and $\mathbf{f}$, respectively. $\mathbf{i}$ The fetus in $\mathbf{e}, \mathbf{f}$, and $\mathbf{h}$ that died in utero prior to the MR scan shown (right) versus a normal E18.5 fetus (left). Tails were removed for genotyping. $\mathbf{j}$ Initial enhancement fraction in vascularized resorped placentas was significantly decreased as compared to regular (C57BL/6 J) and tetraploid (GFP-positive) placentas. Specifically, initial enhancement was decreased in both fully resorped fetuses (fetus absent, $w / o F$ ) and placentas of dead fetuses (fetus present, $w F$ ). Red boxes show resorption data-the left bar graph shows all resorption and the right bar graph shows the two different resorption types. There was no difference in initial enhancement between regular and tetraploid placentas. $p$, placenta; mbv, myometrial blood vessels; $F$, fetus; small arrowheads, fully resorped fetus; large arrowheads, a vascularized resorption site. 

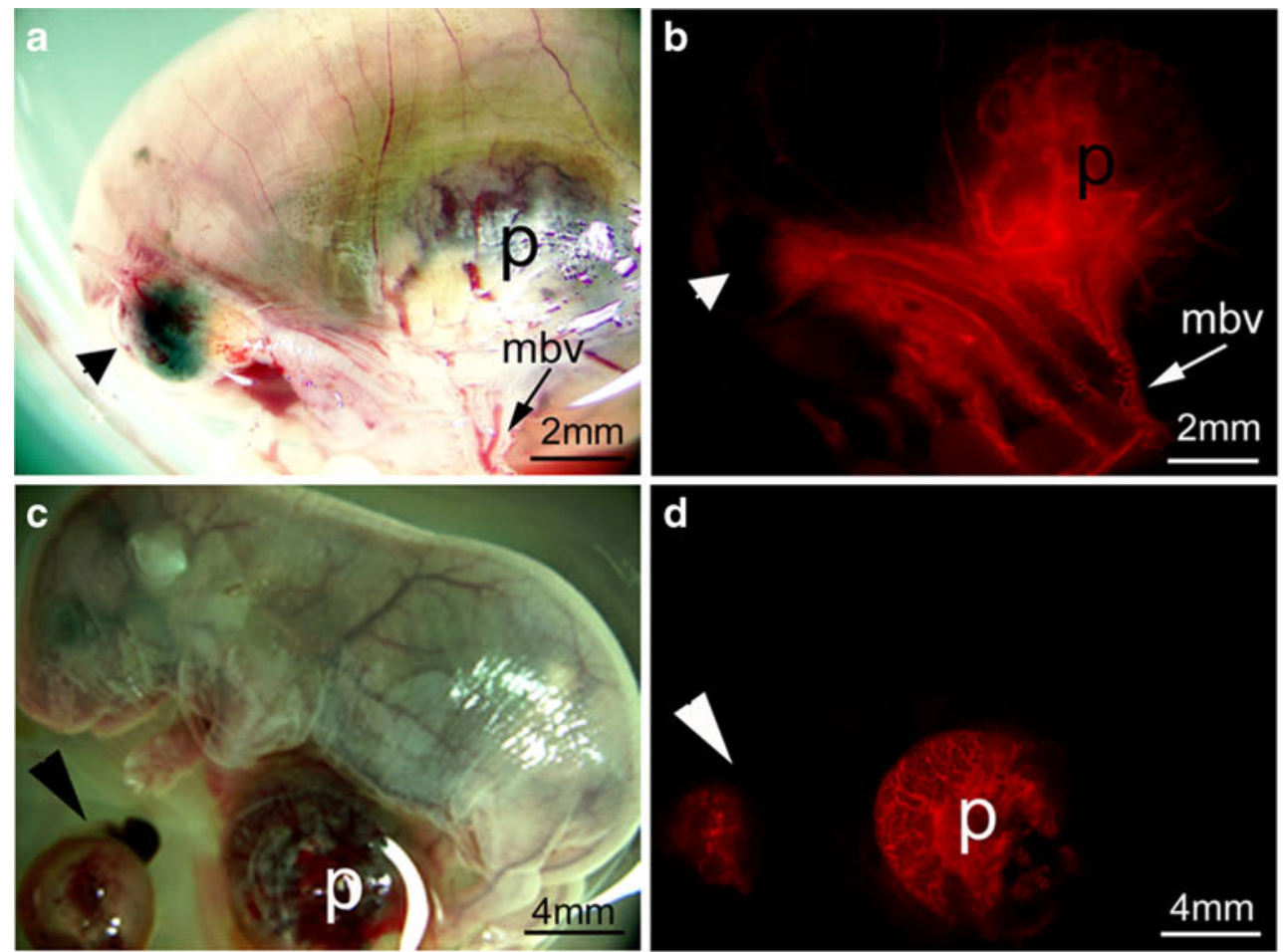

d
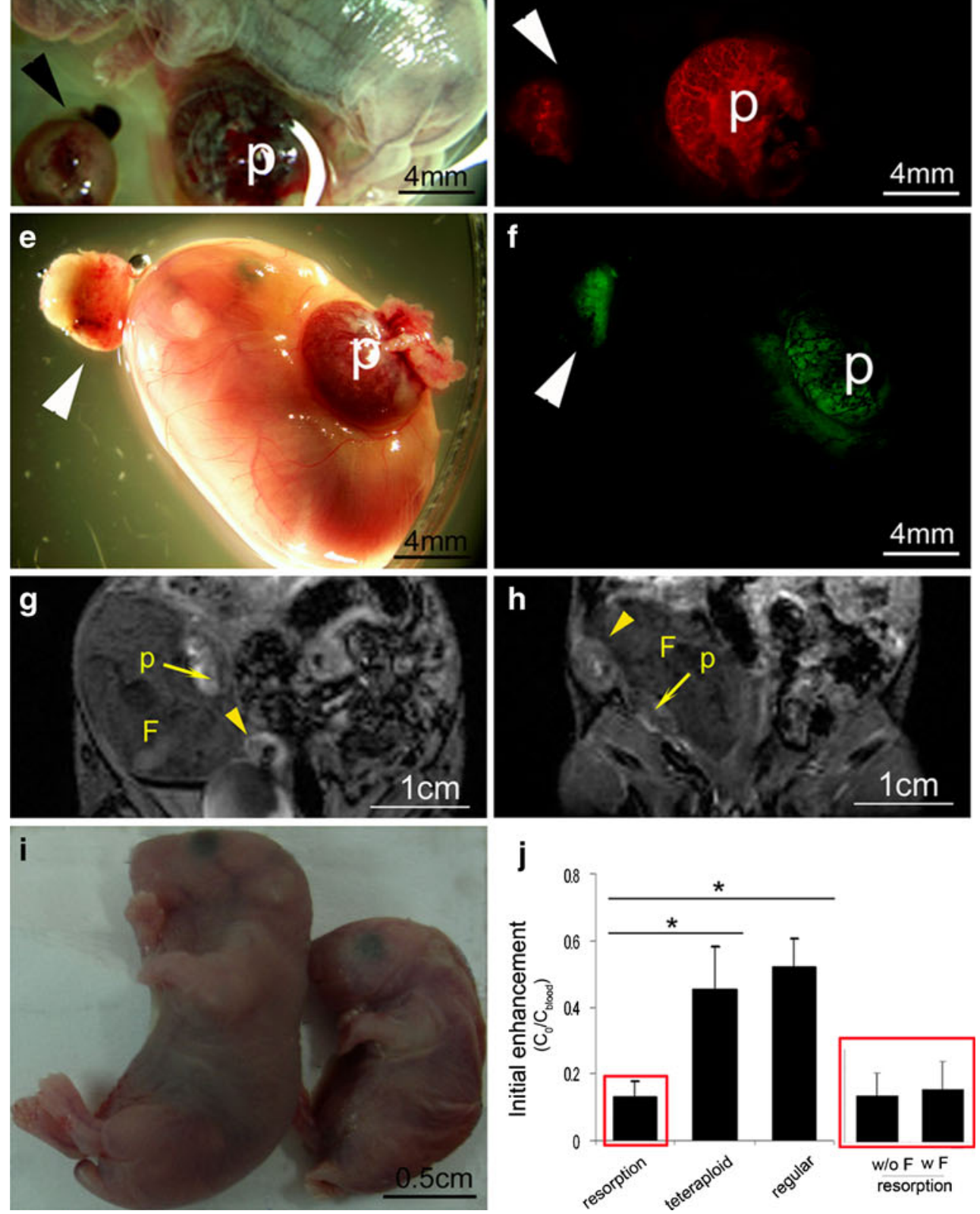
by MRI (see arrowhead in Fig. 5c, d, and g). Hence, using macromolecular DCE-MRI, we were able to non-invasively detect both fully and partially perfused placentas.

In order to independently assess the viability of the placenta, tetraploid complementation was carried out. Using this method, one may generate an embryo of a specific genetic background, bearing a placenta of a different genetic background. In this study, we generated embryos in which only the tetraploid placenta expressed GFP, enabling differentiation of tetraploid placentas from regular placentas. As shown in Fig. 5e and f, an apparently normal tetraploid placenta could be clearly distinguished from a partially resorped placenta.

A major challenge in placental imaging is non-invasive in utero detection of recently resorped fetuses. It will allow the in utero evaluation of the viability of genetically modified mice or the success of manipulations in mouse embryos. In these cases, even though the embryo is dying, it might look normal in the MRI scan or even ex vivo. Fig. 5h presents an MRI image of the same embryos shown in Fig. 5e and f. The placenta of the resorped fetus can be distinguished easily from that of the apparently healthy fetus. However, even though both the unresorped embryo and its placenta appear anatomically normal, an analysis of the MRI data derived from the placenta revealed a significantly lower value for the initial enhancement (Fig. 5h; initial enhancement $=0.06$ for this specific resorped placenta), indicating that both the embryo and its placenta are undergoing resorption. This phenomenon was consistent with significantly reduced initial enhancement values measured for other vascularized resorptions as compared to regular $(\mathrm{C} 57 \mathrm{BL} / 6 \mathrm{~J})$ and tetraploid (GFP-positive) placentas (resorptions: $n=8$ in seven dams; tetraploid: $n=5$ in four dams, $P=0.04$ and B6: $n=4$ in two dams, $P=0.001$; Fig. 5j). Decreased initial enhancement was exhibited in both fully resorped fetuses (fetus absent; w/o F, $n=6$ in five dams) and in placentas of dead fetuses (fetus present; $\mathrm{w} F, n=2$ in two dams).

\section{Discussion}

The placenta is an extra-embryonic tissue that permits the embryo to develop within the female reproductive tract. Considering the increasing interest in phenotypic analysis of genetically modified mice, functional analysis of placental vascularization during pregnancy may serve as an important tool for understanding the role and function of genes. It is currently challenging to evaluate placental circulation in utero in mice [25]. 3D reconstruction of the fetoplacental circulation architecture was reported using vascular corrosion casts [11]. Despite the ability of this method to display the intricate structure of the placenta, data quantification derived from casts may be problematic as superficial vasculature obscures the deeper structures [26]. Stereology of histological sections has been used to obtain quantitative $3 \mathrm{D}$ measures of placental vascularity [24, 27], but sectioning permanently destroys the connectivity between vessels and hence the overall topology. Both corrosion casting and histology provide anatomical structures but cannot be used for longitudinal, functional monitoring of mice in vivo. Micro-CT was shown to provide exquisite images of rat pregnancy [28] as well as enabled 3D visualization and quantification of arterial and venous feto-placental vasculature in the mouse [26]. However, serial dynamic micro-CT imaging would result in biologically unacceptable radiation exposure and thus cannot be applied for in vivo imaging.

Non-invasive imaging of the mouse placenta was also carried out using high-frequency ultrasound. This method was mainly used to generate high-resolution anatomical images of the mouse embryo and placenta from implantation to term [29-31] and to enable the monitoring of changes in hemodynamics of uterine artery, utero- and umbilicoplacental and vitelline circulations [29, 32]. However, both highfrequency and Doppler ultrasound are limited in allowing a direct quantification of the materno-placental perfusion [18].

A few MRI studies were carried out in mice in order to measure tissue blood flow and vascular permeability as well as to characterize the effect of noradrenaline on placental blood flow [19,33]. These studies took advantage of the low molecular weight and small particle diameter of the gadolinium chelate, gadoterate meglumine, in order to investigate the blood flow and placental transport between the mother and its fetuses.

In the current study, we analyzed in utero maternal placental vascularization at different developmental stages. As expected, increase in placental size and vessel density was observed along the pregnancy. Interestingly, whereas a significant increase in placental size was observed by MRI at early stages of the pregnancy (E10.5 and E13.5), no significant difference in the initial enhancement was observed, suggesting that the fetus' demands for nutrients and waste evacuation are adequately compensated by the change in placental size during this period. Only as the pregnancy advances (E13.5 to E18.5) does a remarkable expansion of the maternal blood volume fraction in the placenta take place in order for the fetus to develop normally. In agreement with our MRI data, the histological analysis revealed only a minor difference in the initial enhancement for early developmental stages (for the labyrinth and decidua which are the placental compartments supplying the fetus at this time). A major increase in placental vascularization was observed between the early $(E 10.5,13.5)$ and late (E18.5) stages of the fetus development.

Following the rate of contrast enhancement dynamics, both MRI and fluorescence analysis revealed additional changes in the maternal circulation of the placenta during pregnancy that demonstrate the marked difference of the placenta from other processes of vascular expansion associated with angiogenesis. Angiogenesis is typically characterized by a gradual increase in the concentration of contrast material which is attributed to vascular hyperpermeability, and upon fitting of the data to a two-compartment exchange model it is possible to derive the blood 
volume fraction and the permeability surface area product [15, 34-36]. However, in the late-stage placenta, accumulation of the contrast material occurred without any permeability or extravasation of the contrast material from the maternal circulation to the interstitial space and no transfer of the contrast material to the embryo. Thus, the macromolecular DCE-MRI data could not be analyzed using the available pharmacokinetic models.

In the absence of permeability of the maternal blood spaces in the placenta, dynamic changes in the concentration of the contrast material reflect either its clearance or accumulation within the maternal circulation of the placenta. Significant changes were observed throughout the pregnancy, starting with fast clearance detected at early stages followed by a slow accumulation towards the end of the pregnancy. The delayed elevation in maternal vessel density as compared to the significantly increased ability of the maternal side of the placenta to retain the contrast material at mid-pregnancy (E10.5 to E13.5) may be partially explained by the branching morphogenesis which occurs within the chorioallantoic interface which gives rise to the placental labyrinth [37]. At E13.5, branching morphogenesis has progressed, allowing for a slower outflow of blood from the materno-placental blood spaces although the absolute volume of blood vessels in the forming labyrinth has yet to change. Indeed it was previously reported using stereological methods that the absolute volume of the maternal blood spaces in the labyrinth is rapidly expanding after E13.5, between E14.5 and E16.5 [24]. By the end of pregnancy (E18.5), the labyrinth is fully functioning, as it delivers maximum maternal blood into the placenta and also retains this blood over time to allow an effective exchange of oxygen and nutrients with the fetal blood. In this sense, the functional readout from macromolecular DCE-MRI correlates well with the normal stages of placental development and therefore could aid in the non-invasive evaluation of the impact of impaired placental development during pregnancy.

The rapid clearance of the MRI and fluorescence contrast materials from the maternal circulation at E10.5 and E13.5 was found to be much faster than the clearance from the plasma (vena cava), and this could only be explained by active uptake. Indeed the albumin-based contrast materials are actively internalized by endothelial cells in the decidua and by trophoblast giant cells as well as syncytiotrophoblasts in the labyrinth. The multinucleated syncytiotrophoblasts may serve as a selective barrier or regulator of nutrient, metabolite, gas, waste, and immune interchange between the circulatory systems of mother and fetus [37]. Albumin uptake by trophoblasts by the apical recycling pathway was recently reported for the human placenta $[38,39]$ and albumin could also be taken up by other trophoblast cell types in the mouse placenta such as the sinuosoidal trophoblast giant cells [40].

Intracellular endocytic uptake of biotin-BSA-GdDTPA will differentially affect its detection by MRI and by fluorescence microscopy. As we reported previously, internalization of biotin-BSA-Gd-DTPA into endosomes effectively abolished its $R_{1}$ relaxivity [15, 20, 41, 42]. The localization of internalized albumin conjugated to fluorescence molecules in vacuoles will also decrease the fluorescence signal within the maternal blood space, but the vacuoles will be intensely labeled. The rate of enhancement thus reveals the steady state of maternal albumin between active apical recycling and albumin delivery by the placental circulation.

Dynamic contrast-enhanced MRI allowed us to resolve strain-related differences in embryo transfer procedures and to detect pathological conditions. Analysis of ICR versus B6 embryos revealed a significant difference in their size. ICR embryos were found to be larger than B6 embryos and, accordingly, their placentas were larger as well. Interestingly, these changes in size were not accompanied by a significant difference in either the initial enhancement or rate of enhancement values, although a mild increase in these parameters was observed for the ICR strain. This indicated that the nutritional demands of the two strains are not significantly different.

The utility of macromolecular DCE-MRI for the analysis of impaired placental vascularization was examined as well. Placentas at different stages of resorption were distinguished in utero from healthy placentas, yielding information on the physiological state of the embryo. Specifically, macromolecular DCE-MRI was able to differentiate normal placentas from placentas undergoing resorption, still connected to dead fetuses. These resorping placentas are enhanced after the administration of the contrast media, similarly to normal placentas, but their initial enhancement is similar to that of vascular resorption which has no fetus attached. This MRI methodology may therefore be utilized to follow up on the embryonic physiological state in normal pregnancies in utero as well as in manipulated techniques such as tetraploid complementation and embryo transfer experimentation.

In summary, the data presented here suggests the use of macromolecular DCE-MRI as a tool for in utero phenotypic evaluation of the impact of the fetal genotype on the maternal albumin turnover in the placenta.

Acknowledgement. We thank Dr. Yisrael Parmet and Prof. Stanton A. Glantz for their guidance on the statistical analyses and Nathan Hunkapiller for helpful discussions. This work was supported by the 7th Framework European Research Council Advanced Grant 232640IMAGO. Michal Neeman is incumbent of the Helen and Morris Mauerberger Chair.

Conflicts of interest. None of the authors have a conflict of interest.

Open Access. This article is distributed under the terms of the Creative Commons Attribution Noncommercial License which permits any noncommercial use, distribution, and reproduction in any medium, provided the original author(s) and source are credited. 


\section{References}

1. Austin CP, Battey JF, Bradley A et al (2004) The knockout mouse project. Nat Genet 36(9):921-924

2. Nagy A, Gocza E, Diaz EM et al (1990) Embryonic stem cells alone are able to support fetal development in the mouse. Development 110 (3):815-821

3. Carmeliet P, Ferreira V, Breier G et al (1996) Abnormal blood vessel development and lethality in embryos lacking a single VEGF allele. Nature 380(6573):435-439

4. Hesse M, Watson ED, Schwaluk T, Magin TM (2005) Rescue of keratin 18/19 doubly deficient mice using aggregation with tetraploid embryos. Eur J Cell Biol 84(2-3):355-361

5. Draper JS, Nagy A (2007) Improved embryonic stem cell technologies. Handb Exp Pharmacol 178:107-128

6. Reynolds LP, Redmer DA (2001) Angiogenesis in the placenta. Biol Reprod 64(4):1033-1040

7. Regnault TR, Galan HL, Parker TA, Anthony RV (2002) Placental development in normal and compromised pregnancies-a review. Placenta 23(Suppl A):S119-S129

8. Bassil S, Magritte JP, Roth J, Nisolle M, Donnez J, Gordts S (1995) Uterine vascularity during stimulation and its correlation with implantation in in-vitro fertilization. Hum Reprod 10(6):1497-1501

9. Harrington K, Carpenter RG, Goldfrad C, Campbell S (1997) Transvaginal Doppler ultrasound of the uteroplacental circulation in the early prediction of pre-eclampsia and intrauterine growth retardation. $\mathrm{Br}$ Obstet Gynaecol 104(6):674-681

10. Baergen RN, Malicki D, Behling C, Benirschke K (2001) Morbidity, mortality, and placental pathology in excessively long umbilical cords: retrospective study. Pediatr Dev Pathol 4(2):144-153

11. Adamson SL, Lu Y, Whiteley KJ et al (2002) Interactions between trophoblast cells and the maternal and fetal circulation in the mouse placenta. Dev Biol 250(2):358-373

12. Georgiades P, Ferguson-Smith AC, Burton GJ (2002) Comparative developmental anatomy of the murine and human definitive placentae. Placenta 23(1):3-19

13. Girsh E, Plaks V, Gilad AA et al (2007) Cloprostenol, a prostaglandin F (2alpha) analog, induces hypoxia in rat placenta: BOLD contrast MRI. NMR Biomed 20(1):28-39

14. Gilead A, Meir G, Neeman M (2004) The role of angiogenesis, vascular maturation, regression and stroma infiltration in dormancy and growth of implanted MLS ovarian carcinoma spheroids. Int J Cancer 108 (4):524-531

15. Dafni H, Landsman L, Schechter B, Kohen F, Neeman M (2002) MRI and fluorescence microscopy of the acute vascular response to VEGF165: vasodilation, hyper-permeability and lymphatic uptake, followed by rapid inactivation of the growth factor. NMR Biomed 15 (2):120-131

16. Israely T, Dafni H, Nevo N, Tsafriri A, Neeman M (2004) Angiogenesis in ectopic ovarian xenotransplantation: multiparameter characterization of the neovasculature by dynamic contrast-enhanced MRI. Magn Reson Med 52(4):741-750

17. Plaks V, Kalchenko V, Dekel N, Neeman M (2006) MRI analysis of angiogenesis during mouse embryo implantation. MRM 5(55(5)):10131022

18. Salomon LJ, Siauve N, Balvay D et al (2005) Placental perfusion MR imaging with contrast agents in a mouse model. Radiology 235(1):73-80

19. Salomon LJ, Siauve N, Taillieu F et al (2005) In vivo dynamic MRI measurement of the noradrenaline-induced reduction in placental blood flow in mice. Placenta 25:25

20. Dafni H, Gilead A, Nevo N, Eilam R, Harmelin A, Neeman M (2003) Modulation of the pharmacokinetics of macromolecular contrast material by avidin chase: MRI, optical, and inductively coupled plasma mass spectrometry tracking of triply labeled albumin. Magn Reson Med 50(5):904-914

21. Hadjantonakis AK, Gertsenstein M, Ikawa M, Okabe M, Nagy A (1998) Generating green fluorescent mice by germline transmission of green fluorescent ES cells. Mech Dev 76(1-2):79-90
22. Solter D, Knowles BB (1975) Immunosurgery of mouse blastocyst Proc Natl Acad Sci USA 72(12):5099-5102

23. Nagy A, Gertsenstein M, Vintersten K, Berhringer R (2003) Manipulation of postimplantaion embryos (chapter 5). Manipulation of mouse embryo, 3rd edn. Cold Spring Harbor Laboratory, Cold Spring Harbor, pp 222-223

24. Coan PM, Ferguson-Smith AC, Burton GJ (2004) Developmental dynamics of the definitive mouse placenta assessed by stereology. Biol Reprod 70(6):1806-1813

25. Jansson T, Powell TL (2000) Placental nutrient transfer and fetal growth. Nutrition 16(7-8):500-502

26. Rennie MY, Whiteley KJ, Kulandavelu S, Adamson SL, Sled JG (2007) $3 \mathrm{D}$ visualisation and quantification by microcomputed tomography of late gestational changes in the arterial and venous feto-placental vasculature of the mouse. Placenta 28(8-9):833-840

27. Mayhew TM (2006) Stereology and the placenta: where's the point?-a review. Placenta 27(Suppl A):S17-S25

28. Winkelmann CT, Wise LD (2009) High-throughput micro-computed tomography imaging as a method to evaluate rat and rabbit fetal skeletal abnormalities for developmental toxicity studies. J Pharmacol Toxicol Methods 59(3):156-165

29. Foster FS, Zhang MY, Zhou YQ et al (2002) A new ultrasound instrument for in vivo microimaging of mice. Ultrasound Med Biol 28 (9):1165-1172

30. Slevin JC, Byers L, Gertsenstein M et al (2006) High resolution ultrasound-guided microinjection for interventional studies of early embryonic and placental development in vivo in mice. BMC Dev Biol 6:10

31. Mu J, Slevin JC, Qu D, McCormick S, Adamson SL (2008) In vivo quantification of embryonic and placental growth during gestation in mice using micro-ultrasound. Reprod Biol Endocrinol 6:34

32. Mu J, Adamson SL (2006) Developmental changes in hemodynamics of uterine artery, utero- and umbilicoplacental, and vitelline circulations in mouse throughout gestation. Am J Physiol Heart Circ Physiol 291(3): H1421-H1428

33. Taillieu F, Salomon LJ, Siauve N et al (2006) Placental perfusion and permeability: simultaneous assessment with dual-echo contrastenhanced MR imaging in mice. Radiology 241(3):737-745

34. Bhujwalla ZM, Artemov D, Natarajan K, Ackerstaff E, Solaiyappan M (2001) Vascular differences detected by MRI for metastatic versus nonmetastatic breast and prostate cancer xenografts. Neoplasia 3 (2): $143-153$

35. Tofts PS, Berkowitz BA (1994) Measurement of capillary permeability from the Gd enhancement curve: a comparison of bolus and constant infusion injection methods. Magn Reson Imaging 12(1):81-91

36. Daldrup-Link HE, Okuhata Y, Wolfe A et al (2004) Decrease in tumor apparent permeability-surface area product to a MRI macromolecular contrast medium following angiogenesis inhibition with correlations to cytotoxic drug accumulation. Microcirculation 11(5):387-396

37. Watson ED, Cross JC (2005) Development of structures and transport functions in the mouse placenta. Physiol Bethesda 20:180-193

38. Myllynen PK, Loughran MJ, Howard CV, Sormunen R, Walsh AA, Vahakangas KH (2008) Kinetics of gold nanoparticles in the human placenta. Reprod Toxicol 26(2):130-137

39. Lambot N, Lybaert P, Boom A et al (2006) Evidence for a clathrinmediated recycling of albumin in human term placenta. Biol Reprod 75 (1):90-97

40. Coan PM, Ferguson-Smith AC, Burton GJ (2005) Ultrastructural changes in the interhaemal membrane and junctional zone of the murine chorioallantoic placenta across gestation. J Anat 207(6):783-796

41. Granot D, Addadi Y, Kalchenko V, Harmelin A, Kunz-Schughart LA, Neeman M (2007) In vivo imaging of the systemic recruitment of fibroblasts to the angiogenic rim of ovarian carcinoma tumors. Cancer Res 67(19):9180-9189

42. Granot D, Kunz-Schughart LA, Neeman M (2005) Labeling fibroblasts with biotin-BSA-GdDTPA-FAM for tracking of tumor-associated stroma by fluorescence and MR imaging. Magn Reson Med 54 (4):789-797 